\title{
Cardiometabolic risk factors in young women with macroprolactinaemia
}

\author{
Robert Krysiak', Bogdan Marek2, ${ }^{2}$, Bogusław Okopieńn \\ ${ }^{1}$ Department of Internal Medicine and Clinical Pharmacology, Medical University of Silesia, Katowice, Poland \\ ${ }^{2}$ Division of Pathophysiology, Department of Pathophysiology and Endocrinology, Medical University of Silesia, Zabrze, Poland \\ ${ }^{3}$ Endocrinological Ward, Third Provincial Hospital, Rybnik, Poland
}

\begin{abstract}
Introduction: The predominance of high-molecular-weight forms of prolactin in plasma is referred to as macroprolactinaemia. Unlike monomeric hyperprolactinaemia, no previous study has investigated cardiometabolic risk factors in subjects with elevated macroprolactin content.

Material and methods: We studied two age-, weight-, and blood pressure-matched groups of premenopausal women: 11 women with macroprolactinaemia and 11 women with prolactin levels within the reference range. The outcomes of interest included: glucose homeostasis markers, plasma lipids, as well as plasma levels of uric acid, high-sensitivity C-reactive protein (hsCRP), fibrinogen, homocysteine, and 25-hydroxyvitamin D.

Results: Circulating levels of hsCRP and 2-h postchallenge plasma glucose, as well as the homeostatic model assessment 1 of insulin resistance ratio, were higher, while levels of HDL cholesterol and 25-hydroxyvitamin D were lower in women with macroprolactinaemia than in women without elevated levels of prolactin. In women with elevated levels of big-big prolactin, values of hsCRP and 25-hydroxyvitamin D correlated with the degree of insulin resistance and, similarly to HDL cholesterol and 2-h postchallenge plasma glucose, with macroprolactin content. There were no differences between the study groups in concentrations of fasting glucose, total cholesterol, LDL cholesterol, triglycerides, uric acid, fibrinogen, and homocysteine.

Conclusions: The obtained results indicate that young women with macroprolactinaemia seem to be characterised by slightly increased cardiometabolic risk. (Endokrynol Pol 2019; 70 (4): 336-341)
\end{abstract}

Key words: cardiovascular risk; macroprolactin; monomeric prolactin; risk factors

\section{Introduction}

Apart from monomeric prolactin, being the most abundant (60-90\%) and biologically active form of prolactin, this hormone occurs in the circulation in two other molecular forms: big-prolactin, consisting of prolactin dimers, and big-big prolactin, also referred to as macroprolactin. Macroprolactin comprises mainly high-molecular-mass complexes of $23 \mathrm{kDa}$ prolactin and $\mathrm{IgG}$ autoantibodies [1-3]. Less frequently occurring forms of big-big prolactin include: complexes of prolactin with IgA or IgM, highly glycosylated monomeric prolactin, as well as covalent or non-covalent aggregates of monomeric prolactin [4, 5]. Macroprolactinaemia, a condition associated with markedly increased circulating big-big prolactin levels, is usually diagnosed if more than $60 \%$ of total prolactin is precipitated by polyethylene glycol [5]. The overall prevalence of elevated macroprolactin content in the general population ranges between $3 \%$ and $4 \%$, but increases up to $35 \%$ in patients with elevated prolactin levels [3].
Long-term excess of monomeric prolactin is often associated with impaired glucose tolerance, hyperinsulinaemia, insulin resistance, atherogenic dyslipidaemia, subclinical atherosclerosis, endothelial dysfunction, and weight gain [6-13]. Dopamine agonists, the drugs of choice in the treatment of monomeric hyperprolactinaemia, reduced plasma levels of cardiometabolic risk factors [14]. Moreover, cardiometabolic effects of atorvastatin [15] and fenofibrate [16] depended on the prolactin status of patients, being much less pronounced (or even absent) in women with untreated hyperprolactinaemia than in women with bromocriptine-treated hyperprolactinaemia and drug-naïve women with normal prolactin levels. These results suggest that the presence of elevated levels of monomeric prolactin eliminates the beneficial effect of atorvastatin on plasma levels of cardiometabolic risk factors.

Although macroprolactinaemia is generally regarded as a benign condition and most subjects remain asymptomatic $[2,4]$, some authors disagree with this point of view. Gibney et al. [17] observed 
that a significant proportion of women with elevated content of big-big prolactin developed galactorrhoea, oligomenorrhoea/amenorrhoea, and subfertility. In turn, Krysiak et al. [18] found that young women with isolated macroprolactinaemia were characterised by abnormal sexual desire and depressive symptoms. These disturbances, less pronounced than in women with monomeric hyperprolactinaemia [18], suggest that macroprolactinaemia should be taken into consideration in all women with elevated prolactin levels, who complain of infertility, menstrual disturbances, or hypolipidaemia. To the best of our knowledge, no previous study investigated cardiometabolic risk in patients with macroprolactinaemia. However, Gulcelik et al. [19] observed that macroprolactin occurs much more frequently in patients with diabetes than in subjects without carbohydrate metabolism disturbances, while levels of glycated haemoglobin were higher in diabetic patients with macroprolactinaemia than in individuals with diabetes but without elevated macroprolactin content. Therefore, the current study was aimed at comparing plasma lipids, glucose homeostasis markers and circulating levels of uric acid, high-sensitivity C-reactive protein (hsCRP), fibrinogen, homocysteine, and 25-hydroxyvitamin $\mathrm{D}$, regarded as well-established cardiometabolic risk factors [20-26], between young women with macroprolactinaemia and their healthy counterparts.

\section{Material and methods}

The participants of the study $(\mathrm{n}=11)$ were recruited among asymptomatic women (20-45 years old) with elevated levels of total prolactin. To be admitted to the study, they had to meet the following criteria of macroprolactinaemia: (a) total prolactin levels above $40 \mathrm{ng} / \mathrm{mL}$ found on two different occasions; (b) prolactin recovery less than $40 \%$, and (c) post-polyethylene glycol circulating prolactin levels less than $25 \mathrm{ng} / \mathrm{mL}$. The study also enrolled 11 age- and weight-matched healthy women with prolactin and macroprolactin levels within the reference range. We excluded patients with coexisting prolactinomas or other pituitary tumours, impaired renal or hepatic function, thyroid disorders, diabetes, acute and chronic inflammatory processes, cardiovascular disease, pregnant or breastfeeding women, as well as patients treated with any drugs. The study was performed in accordance with the 1964 Helsinki Declaration, and the study protocol was accepted by the local Ethics Committee. All women gave written, informed consent to participate in the study.

Venous blood samples were drawn from the antecubital vein between 8.00 and 9.00 a.m., at least $12 \mathrm{~h}$ after the last meal, in a quiet, temperature-controlled room $\left(24-25^{\circ} \mathrm{C}\right)$, in order to avoid circadian fluctuations of the parameters studied. Moreover, glucose levels were measured in samples obtained $2 \mathrm{~h}$ after the oral ingestion of $75 \mathrm{~g}$ of glucose. Fasting and 2-h postchallenge plasma glucose, total cholesterol, high-density lipoprotein (HDL) cholesterol, low-density lipoprotein (LDL) cholesterol, triglycerides, and uric acid were measured with standard methods using commercial kits (Roche Diagnostics, Basel, Switzerland). Plasma levels of prolactin, insulin, and homocysteine were assayed by direct chemiluminescence using acridinium ester technology (ADVIA Centaur XP Immunoassay System, Siemens Healthcare Diagnostics,
Munich, Germany). Plasma levels of 25-hydroxyvitamin D were detected by competitive immunoassay using and a multichannel automatic analyser (Roche Cobas e 411, Mannheim, Germany) and commercially available Roche Diagnostic kits. High-sensitivity C-reactive protein (hsCRP) was measured by immunoassay with chemiluminescent detection (Immulite 2000XPi, Siemens Healthcare, Warsaw, Poland). Fibrinogen levels were measured by the Clauss technique, using an automated BCS XP analyser (Siemens Healthcare, Warsaw, Poland). The homeostatic model assessment 1 of insulin resistance (HOMA1-IR) was calculated as follows: fasting glucose $(\mathrm{mg} / \mathrm{dL}) \times$ fasting insulin $(\mathrm{mIU} / \mathrm{L}) / 405$.

In order to estimate macroprolactin content, equal volumes (250 $\mu \mathrm{L}$ ) of plasma and $25 \%$ cold polyethylene glycol 6000 dissolved in phosphate-buffered saline (Sigma, $137 \mathrm{mmol} / \mathrm{L}$ sodium chloride, $10 \mathrm{mmol} / \mathrm{L}$ sodium phosphate, $\mathrm{pH}=7.4$ ) were mixed in the ratio of $1: 1$ and incubated for $10 \mathrm{~min}$. After vortex mixing for $30 \mathrm{~s}$, the suspension was clarified by centrifugation at $3000 \mathrm{rpm}$ for $30 \mathrm{~min}$. To calculate prolactin recovery, the following equation was used: plasma prolactin after polyethylene glycol precipitation/plasma prolactin before polyethylene glycol precipitation $\times 100$. The post-polyethylene glycol prolactin concentration was determined by multiplying the prolactin result by two in order to correct for the dilution with polyethylene glycol. Macroprolactinaemia was diagnosed if the prolactin recovery was below $40 \%$.

Owing to the skewed distributions, values for prolactin, hsCRP fibrinogen, 25-hydroxyvitamin D, homocysteine, triglycerides, and HOMA1-IR were natural-log transformed to achieve normality and homogeneity of variance. Comparisons between the groups were performed using Student's $t$-test for independent samples. Categorical variables were analysed by $\chi^{2}$ test. Correlations between the measured variables were calculated using Pearson's $r$-tests. The clinical importance of the result was assessed based on the 95\% confidence interval. Differences were described as statistically significant if $95 \%$ confidence intervals did not include the null value and/or two-tailed $\mathrm{p}$ values were less than 0.05 .

\section{Results}

There were no differences between the study groups in terms of age, smoking, body mass index, waist circumference, and systolic and diastolic blood pressure. Both groups differed in levels of prolactin before polyethylene glycol precipitation and macroprolactin content. Compared to women with prolactin levels within the reference range, subjects with macroprolactinaemia had higher plasma levels of hsCRP, higher 24-h postchallenge plasma levels of glucose, and a higher value of HOMA1-IR, as well as lower plasma levels of HDL cholesterol and 25-hydroxyvitamin D. There were no differences between the study groups in concentrations of fasting glucose, uric acid, total cholesterol, LDL cholesterol, triglycerides, fibrinogen, and homocysteine (Tab. I).

In women with macroprolactinaemia circulating levels of hsCRP and 25-hydroxyvitamin D correlated with: levels of prolactin before polyethylene glycol precipitation, content of macroprolactin, HOMA1-IR, as well as with concentrations of HDL cholesterol and 2-h postchallenge plasma glucose (Tab. II). Moreover, levels of prolactin before polyethylene glycol precipitation and content of macroprolactin correlated with HOMA1-IR $(\mathrm{r}=0.29, \mathrm{p}<0.05$ and $\mathrm{r}=0.31, \mathrm{p}<0.05$, 
Table I. Comparison between young women with macroprolactinaemia and their counterparts with prolactin levels within the reference range

\begin{tabular}{|c|c|c|c|}
\hline & $\begin{array}{l}\text { Women } \\
\text { with macroprolactinaemia }\end{array}$ & Control women & $\begin{array}{l}\text { Difference } \\
{[95 \% \mathrm{Cl}]}\end{array}$ \\
\hline Number of patients & 11 & 11 & - \\
\hline Age [years; mean (SD)] & $34(7)$ & $32(7)$ & $-2[-8,4]$ \\
\hline Smoking (\%) & 27 & 36 & - \\
\hline Body mass index [kg/m²; mean (SD)] & $27.1(4.8)$ & $26.9(4.6)$ & $-0.2[-4.4,4.0]$ \\
\hline Waist circumference [cm; mean (SD)] & $88(15)$ & $86(13)$ & $-2[-15,11]$ \\
\hline Systolic blood pressure [mm Hg; mean (SD)] & $134(13)$ & $132(15)$ & $-2[-14,10]$ \\
\hline Diastolic blood pressure [mm Hg; mean (SD)] & $86(6)$ & $85(6)$ & $-1[-6,4]$ \\
\hline $\begin{array}{l}\text { Prolactin before polyethylene glycol precipitation } \\
\text { [ng/mL; mean (SD)] }\end{array}$ & $70(18)$ & $13(6)$ & $-57[-69,-45]^{*}$ \\
\hline $\begin{array}{l}\text { Prolactin after polyethylene glycol precipitation } \\
{[\mathrm{ng} / \mathrm{mL} ; \text { mean (SD)] }}\end{array}$ & $14(7)$ & $12(5)$ & $-2[-7,3]$ \\
\hline Macroprolactin [\%, mean (SD)] & $80(10)$ & $7(5)$ & $-73[-80,-66]^{*}$ \\
\hline Total cholesterol [mg/dL; mean (SD)] & $219(29)$ & $217(32)$ & $-2[-29,25]$ \\
\hline LDL-cholesterol [mg/dL; mean (SD)] & $123(20)$ & $119(18)$ & $-4[-21,13]$ \\
\hline HDL-cholesterol [mg/dL; mean (SD)] & $50(7)$ & $56(6)$ & $6[1,11]^{*}$ \\
\hline Triglycerides [mg/dL; mean (SD)] & $208(48)$ & $185(39)$ & $-23[-62,16]$ \\
\hline Fasting glucose [mg/dL; mean (SD)] & $91(8)$ & $90(8)$ & $-1[-8,6]$ \\
\hline 2-h postchallenge glucose [mg/dL; mean (SD)] & $137(18)$ & $122(15)$ & $-15[-29,-1]^{*}$ \\
\hline HOMA1-IR [mean (SD)] & $2.6(0.8)$ & $1.8(0.7)$ & $-0.8[-1.5,-0.1]^{*}$ \\
\hline Uric acid $[\mu \mathrm{mol} / \mathrm{L} ;$ mean $(\mathrm{SD})]$ & $367(76)$ & $344(69)$ & $-23[-88,42]$ \\
\hline hsCRP [mg/L; mean (SD)] & $2.3(0.8)$ & $1.3(0.6)$ & $-1.0[-1.6,-0.4]^{*}$ \\
\hline Fibrinogen [mg/dL; mean (SD)] & $354(68)$ & $325(80)$ & $-29[-95,37]$ \\
\hline Homocysteine $[\mu \mathrm{mol} / \mathrm{L} ;$ mean $(\mathrm{SD})]$ & $15(7)$ & $13(8)$ & $-2[-9,5]$ \\
\hline 25-hydroxyvitamin D [ng/mL; mean (SD)] & $25(7)$ & $32(8)$ & $7[1,13]^{*}$ \\
\hline
\end{tabular}

*statistically significant difference between both groups. HDL — high-density lipoprotein; HOMA1-IR — the homeostatic model assessment 1 of insulin resistance ratio; hsCRP — high-sensitivity C-reactive protein; LDL — low-density lipoprotein; SD — standard deviation; $\mathrm{Cl}$ — confidence interval

Table II. Correlations between the assessed variables in young women with macroprolactinaemia

\begin{tabular}{|c|c|c|c|c|c|}
\hline & hsCRP & Fibrinogen & Homocysteine & 25-hydroxyvitamin D & Uric acid \\
\hline $\begin{array}{l}\text { Prolactin before polyethylene glycol } \\
\text { precipitation }\end{array}$ & $0.37^{* *}$ & 0.16 & 0.12 & $-0.37^{* *}$ & 0.19 \\
\hline $\begin{array}{l}\text { Prolactin after polyethylene glycol } \\
\text { precipitation }\end{array}$ & 0.22 & 0.20 & 0.15 & -0.19 & 0.18 \\
\hline Macroprolactin & $0.40^{* * *}$ & 0.21 & 0.10 & $-0.34^{* *}$ & 0.11 \\
\hline HOMA1-IR & $0.35^{* *}$ & 0.23 & 0.11 & $-0.27^{*}$ & 0.13 \\
\hline 2-h postchallenge plasma glucose & $0.30^{*}$ & 0.17 & 0.07 & $-0.30^{*}$ & 0.20 \\
\hline HDL-cholesterol & $-0.32^{*}$ & -0.17 & -0.03 & $0.26 *$ & -0.22 \\
\hline
\end{tabular}

Data represent the correlation coefficients (r values). ${ }^{*} p<0.05,{ }^{* *} p<0.01,{ }^{* * *} p<0.001$. HDL — high-density lipoprotein; HOMA1-IR - the homeostatic model assessment 1 of insulin resistance ratio; hsCRP — high-sensitivity C-reactive protein

respectively), HDL cholesterol $(\mathrm{r}=-0.31, \mathrm{p}<0.05$ and $\mathrm{r}=-0.25, \mathrm{p}<0.05$, respectively), and 2 -h postchallenge plasma glucose $(r=0.26, p<0.05$ and $r=0.32, p<0.05$, respectively). In both study groups, there were correlations between HOMA1-IR and 2-h postchallenge plasma glucose (women with macroprolactinaemia: $\mathrm{r}=0.41, \mathrm{p}<0.001$; women without macroprolactinaemia: $\mathrm{r}=0.43, \mathrm{p}<0.001$ ), HDL cholesterol (women with macroprolactinaemia: $\mathrm{r}=-0.35, \mathrm{p}<0.01$; women without macroprolactinaemia: $r=-0.38, p<0.01$ ) and 
triglycerides (women with macroprolactinaemia: $\mathrm{r}=0.38, \mathrm{p}<0.01$; women without macroprolactinaemia: $r=0.40, p<0.001$ ) (data not shown). No other correlations were found.

\section{Discussion}

This study has shown for the first time that the presence of macroprolactinaemia in apparently healthy young women was associated with abnormal levels of some cardiometabolic risk factors: hsCRP, 2-h postchallenge glucose, HDL cholesterol, and 25-hydroxyvitamin D, as well with an increased value of HOMA1-IR, suggesting that macroprolactinaemia may slightly increase the risk of development of cardiovascular and metabolic disorders. This risk seems to be less prominent than in patients with either untreated or treatment-resistant monomeric hyperprolactinaemia, who were characterised by abnormally low levels of 25-hydroxyvitamin $\mathrm{D}$, as well as increased circulating concentrations of insulin-like growth factor-1, free fatty acids, uric acid, hsCRP, homocysteine, and fibrinogen [14]. The participants of the current study were individuals with selectively increased macroprolactin content in whom plasma levels of monomeric prolactin within the reference range. Therefore, the study protocol allowed us to eliminate the possibility that the observed changes were secondary to true hyperprolactinaemia coexisting with increased production of big-big prolactin. The presence of such coexistences was previously described by other research teams $[27,28]$. Taking into account that disturbances in hsCRP, 2-h postchallenge glucose, HDL cholesterol, and 25-hydroxyvitamin D contribute to the development of atherosclerosis and diabetes, as well as their complications [20-22, 26], the obtained results may be clinically significant. Our findings, being in agreement with previous observations $[17,18]$, suggest that elevated levels of big-big prolactin should not be considered as a benign condition without pathogenic relevance.

All markers, the level of which differed between patients with macroprolactinaemia and healthy subjects, correlated with plasma levels of prolactin before polyethylene glycol precipitation and content of macroprolactin, but not with plasma levels of prolactin after polyethylene glycol precipitation. The presence of these correlations indicates that this relationship does not seem to be accidental, suggesting that abnormal levels of cardiometabolic risk factors are probably directly linked to elevated levels of big-big prolactin. It is possible that low vitamin D status, reflected in the present study by low levels of 25-hydroxyvitamin D, may contribute to the pathogenesis of macroprolactinaemia. We think so because exogenous preparations of vitamin D administered for four months reduced big-big prolactin content [29].

Circulating levels of hsCRP and vitamin D correlated with HOMA1-IR, 2-h postchallenge plasma glucose, and HDL cholesterol, being the markers of insulin receptor action [20,21]. Based on the obtained results, it may be assumed that low-grade systemic inflammation and hypovitaminosis $\mathrm{D}$ develop as a consequence of carbohydrate metabolism abnormalities, or their presence may disturb glucose homeostasis. Similar relationships were observed previously in patients with monomeric hyperprolactinaemia, the characteristic features of which were hyperinsulinaemia, insulin resistance, atherogenic dyslipidaemia, and weight gain [14].

Two different molecular mechanisms may explain the obtained results. According to the first explanation put forward by other authors [17], high molecular mass complexes of big-big prolactin may dissociate in peripheral tissues releasing monomeric prolactin. This possibility is particularly probable in the case of the commonest form of macroprolactin, namely complexes consisting of monomeric prolactin and low-affinity, high-capacity $\operatorname{IgG}[1,2]$. Disturbances in cardiometabolic risk factors, which are less expressed than in subjects with true hyperprolactinaemia may result from the fact that only a small percentage of macroprolactin dissociates, and the excess of monomeric prolactin is less expressed than in subjects with monomeric hyperprolactinaemia. In line with an alternative explanation, big-big prolactin may have a week affinity to prolactin receptors. However, taking into account the prolonged clearance rate $[2,4]$, macroprolactin may chronically stimulate prolactin receptors, exerting prolactin-like effects at the level of different peripheral tissues.

Although milder than in true hyperprolactinaemia, cardiometabolic abnormalities in women with elevated macroprolactin content may be much more difficult to treat. The only agent found to slightly reduce macroprolactin content is exogenous vitamin D [29]. Unlike elevated levels of monomeric prolactin, which are easy to treat with dopamine agonists, particularly with cabergoline [14], the reduction in big-big prolactin content was observed only in some subjects with macroprolactinaemia [5, 30]. Moreover, testosterone [31] and metformin [32] did not affect macroprolactin levels (although metformin was found to reduce concentrations of monomeric prolactin [33, 34]), while ethinyl estradiol plus levonorgestrel led to an increase in its content [35].

We are aware of some limitations of our study. The most important of them is a small number of participants. Secondly, the study did not investigate clinical outcomes, particularly morbidity or mortality. Moreover, because the study included only women, it is not 
certain whether cardiometabolic risk is increased in men with macroprolactinaemia. Finally, the study protocol does not allow us to conclude whether elevated macroprolactin content increases cardiometabolic risk in patients with coexistent cardiovascular or metabolic disorders.

\section{Conclusions}

Compared to healthy counterparts with plasma prolactin levels within the reference range, women with macroprolactinaemia had increased plasma levels of hsCRP and 2-h postload glucose, an increased value of HOMA1-IR, as well as reduced plasma levels of HDL cholesterol and 25-hydroxyvitamin D. Macroprolactinaemia-induced changes in hsCRP and 25-hydroxyvitamin $\mathrm{D}$ that are correlated with the degree of insulin resistance and, similarly to HDL cholesterol and 2-h postchallenge plasma glucose, with macroprolactin content. The obtained results indicate that young women with macroprolactinaemia may be characterised by slightly increased cardiometabolic risk.

\section{Conflict of interest}

The authors declare no conflict of interest.

\section{Acknowledgements}

This work was not supported by any external source of funding. The experiments comply with the current law of Poland.

\section{Funding}

This work was not supported by any external source of funding.

\section{References}

1. Kasum M, Orešković S, Čehić E, et al. Laboratory and clinical significance of macroprolactinemia in women with hyperprolactinemia. Taiwan J Obstet Gynecol. 2017; 56(6): 719-724, doi: 10.1016/j.tjog.2017.10.002, indexed in Pubmed: 29241908.

2. Fahie-Wilson M, Smith TP. Determination of prolactin: the macroprolactin problem. Best Pract Res Clin Endocrinol Metab. 2013; 27(5): 725-742, doi: 10.1016/j.beem.2013.07.002, indexed in Pubmed: 24094642.

3. Shimatsu A, Hattori N. Macroprolactinemia: diagnostic, clinical, and pathogenic significance. Clin Dev Immunol. 2012; 2012: 167132, doi: 10.1155/2012/167132, indexed in Pubmed: 23304187.

4. Fahie-Wilson MN, John R, Ellis AR. Macroprolactin; high molecular mass forms of circulating prolactin. Ann Clin Biochem. 2005; 42(Pt 3): 175-192, doi: 10.1258/0004563053857969, indexed in Pubmed: 15949152.

5. Vallette-Kasic S, Morange-Ramos I, Selim A, et al. Macroprolactinemia revisited: a study on 106 patients. J Clin Endocrinol Metab. 2002; 87(2): 581-588, doi: 10.1210/jcem.87.2.8272, indexed in Pubmed: 11836289.

6. dos Santos Silva CM, Barbosa FRP, Lima GAB, et al. BMI and metabolic profile in patients with prolactinoma before and after treatment with dopamine agonists. Obesity (Silver Spring). 2011; 19(4): 800-805, doi: 10.1038/oby.2010.150, indexed in Pubmed: 20559294.

7. Yavuz D, Deyneli O, Akpinar I, et al. Endothelial function, insulin sensitivity and inflammatory markers in hyperprolactinemic pre-menopausal women. Eur J Endocrinol. 2003; 149(3): 187-193, indexed in Pubmed: 12943520.

8. Berinder K, Nyström T, Höybye C, et al. Insulin sensitivity and lipid profile in prolactinoma patients before and after normalization of pro- lactin by dopamine agonist therapy. Pituitary. 2011; 14(3): 199-207, doi: 10.1007/s11102-010-0277-9, indexed in Pubmed: 21128120.

9. Serri O, Li L, Mamputu JC, et al. The influences of hyperprolactinemia and obesity on cardiovascular risk markers: effects of cabergoline therapy. Clin Endocrinol (Oxf). 2006; 64(4): 366-370, doi: 10.1111/j.136 5-2265.2006.02469.x, indexed in Pubmed: 16584506.

10. Jiang XB, He DS, Mao ZG, et al. BMI, apolipoprotein B/apolipoprotein A-I ratio, and insulin resistance in patients with prolactinomas: a pilot study in a Chinese cohort. Tumour Biol. 2013; 34(2): 1171-1176, doi: 10.1007/s13277-013-0660-z, indexed in Pubmed: 23345015.

11. Jiang $\mathrm{XB}, \mathrm{Li} C \mathrm{CL}, \mathrm{He} \mathrm{DS}$, et al. Increased carotid intima media thickness is associated with prolactin levels in subjects with untreated prolactinoma: a pilot study. Pituitary. 2014; 17(3): 232-239, doi: 10.1007/s11102-013-0495-z, indexed in Pubmed: 23756783.

12. Greenman $Y$, Tordjman K, Stern N. Increased body weight associated with prolactin secreting pituitary adenomas: weight loss with normalization of prolactin levels. Clin Endocrinol (Oxf). 1998; 48(5): 547-553, indexed in Pubmed: 9666865.

13. Schmid C, Goede DL, Hauser RS, et al. Increased prevalence of high Body Mass Index in patients presenting with pituitary tumours: severe obesity in patients with macroprolactinoma. Swiss Med Wkly. 2006; 136(15-16): 254-258, doi: 2006/15/smw-10955, indexed in Pubmed: 16708311.

14. KrysiakR, Okopien B. Different effects of cabergoline and bromocriptine on metabolic and cardiovascular risk factors in patients with elevated prolactin levels. Basic Clin Pharmacol Toxicol. 2015; 116(3): 251-256, doi: 10.1111/bcpt.12307, indexed in Pubmed: 25123447.

15. Krysiak R, Szkróbka W, Okopień B. Different effects of atorvastatin on cardiometabolic risk factors in young women with and without hyperprolactinemia. J Clin Pharmacol. 2019; 59(1): 83-89, doi: 10.1002/jcph.1301, indexed in Pubmed: 30129670.

16. Krysiak R, Szkróbka W, Okopień B. Different effects of fenofibrate on cardiometabolic risk factors in young women with and without hyperprolactinemia. Pharmacol Rep. 2019; 71(1): 61-66, doi: 10.1016/j. pharep.2018.09.004, indexed in Pubmed: 30469130.

17. Gibney J, Smith TP, McKenna TJ. Clinical relevance of macroprolactin. Clin Endocrinol (Oxf). 2005; 62(6): 633-643, doi: 10.1111/j.1365-2265.200 5.02243.x, indexed in Pubmed: 15943822.

18. Krysiak R, Drosdzol-Cop A, Skrzypulec-Plinta V, et al. Sexual function and depressive symptoms in young women with elevated macroprolactin content: a pilot study. Endocrine. 2016; 53(1): 291-298, doi: 10.1007/s12020-016-0898-5, indexed in Pubmed: 26902871.

19. Gulcelik NE, Usman A. Macroprolactinaemia in diabetic patients. Neuro Endocrinol Lett. 2010; 31(2): 270-274, indexed in Pubmed: 20424586.

20. Dyaczyński M, Scanes CG, Koziec H, et al. Endocrine implications of obesity and bariatric surgery. Endokrynol Pol. 2018; 69(5): 574-597, doi: 10.5603/EP.2018.0059, indexed in Pubmed: 30379322.

21. Gilowski W, Krysiak R, Marek B, et al. The effect of short-term perindopril and telmisartan treatment on circulating levels of anti-inflammatory cytokines in hypertensive patients. Endokrynol Pol. 2018; 69(6): 667-674, doi: 10.5603/EP.a2018.0068, indexed in Pubmed: 30259507.

22. Kinlay S, Egido J. Inflammatory biomarkers in stable atherosclerosis. Am J Cardiol. 2006; 98(11A): 2P_8P, doi: 10.1016/j.amjcard.2006.09.014, indexed in Pubmed: 17126676.

23. Feig DI, Kang DH, Johnson RJ. Uric acid and cardiovascular risk. N Engl J Med. 2008; 359(17): 1811-1821, doi: 10.1056/NEJMra0800885, indexed in Pubmed: 18946066.

24. Krysiak R, Okopień B, Herman Z. Effects of HMG-CoA reductase inhibitors on coagulation and fibrinolysis processes. Drugs. 2003; 63(17): 1821-1854, doi: 10.2165/00003495-200363170-00005, indexed in Pubmed: 12921488 .

25. McCully KS. Homocysteine, vitamins, and vascular disease prevention. Am J Clin Nutr. 2007; 86(5): 1563S-8S, doi: 10.1093/ajcn/86.5.1563S, indexed in Pubmed: 17991676.

26. Wang Lu, Song Y, Manson JE, et al. Circulating 25-hydroxy-vitamin D and risk of cardiovascular disease: a meta-analysis of prospective studies. Circ Cardiovasc Qual Outcomes. 2012; 5(6): 819-829, doi: 10.1161/CIRCOUTCOMES.112.967604, indexed in Pubmed: 23149428.

27. Mounier C, Trouillas J, Claustrat B, et al. Macroprolactinaemia associated with prolactin adenoma. Hum Reprod. 2003; 18(4): 853-857, indexed in Pubmed: 12660284

28. Tamer G, Telci A, Mert M, et al. Prevalence of pituitary adenomas in macroprolactinemic patients may be higher than it is presumed. Endocrine. 2012; 41(1): 138-143, doi: 10.1007/s12020-011-9536-4, indexed in Pubmed: 21959531.

29. Krysiak R, Kowalska B, Szkróbka W, et al. The association between macroprolactin levels and vitamin D status in premenopausal women with macroprolactinemia: a pilot study. Exp Clin Endocrinol Diabetes. 2015; 123(8): 446-450, doi: 10.1055/s-0035-1555877, indexed in Pubmed: 26179930.

30. Olukoga AO. Macroprolactinemia is clinically important. J Clin Endocrinol Metab. 2002; 87(10): 4833-4; author reply 4834, doi: 10.1210/jc.2002-020936, indexed in Pubmed: 12364483. 
31. Krysiak R, Kowalska B, Szkróbka W, et al. A neutral effect of testosterone therapy on macroprolactin content in men with mac roprolactinemia and late-onset hypogonadism. Pharmacol Rep. 2016; 68(1): 139-143, doi: 10.1016/j.pharep.2015.08.003, indexed in Pubmed: 26721365

32. Krysiak R, Szkróbka W, Okopień B. A Neutral Effect of Metformin Treatment on Macroprolactin Content in Women with Macroprolactinemia. Exp Clin Endocrinol Diabetes. 2017; 125(4): 223-228, doi: 10.1055/s-0042-117718, indexed in Pubmed: 27750350.

33. Zheng $\mathrm{W}$, Yang $\mathrm{XH}$, Cai DB, et al. Adjunctive metformin for antipsychotic-related hyperprolactinemia: A meta-analysis of ran- domized controlled trials. J Psychopharmacol. 2017; 31(5): 625-631, doi: 10.1177/0269881117699630, indexed in Pubmed: 28372526.

34. Krysiak R, Okrzesik J, Okopien B. The effect of short-term metformin treatment on plasma prolactin levels in bromocriptine-treated patients with hyperprolactinaemia and impaired glucose tolerance: a pilot study. Endocrine. 2015; 49(1): 242-249, doi: 10.1007/s12020-014-0428-2, indexed in Pubmed: 25239203.

35. Krysiak R, Kowalska B, Szkróbka W, et al. The effect of oral contraception on macroprolactin levels in women with macroprolactinemia: A pilot study. Pharmacol Rep. 2015; 67(5): 854-857, doi: 10.1016/j. pharep.2015.02.001, indexed in Pubmed: 26398376. 